\title{
Why are behaviors of children suffering from various neuronopathic types of mucopolysaccharidoses different?
}

\author{
Grzegorz Węgrzyn a,*, Joanna Jakóbkiewicz-Banecka a , Magdalena Narajczyk ${ }^{\mathrm{a}}$, Andrzej Wiśniewski ${ }^{\mathrm{b}}$, \\ Ewa Piotrowska ${ }^{a}$, Magdalena Gabig-Cimińska ${ }^{c}$, Anna Kloska ${ }^{a}$, Monika Słomińska-Wojewódzka ${ }^{a}$, \\ Anna Korzon-Burakowska ${ }^{\mathrm{d}}$, Alicja Węgrzyn ${ }^{\mathrm{c}}$ \\ ${ }^{a}$ Department of Molecular Biology, University of Gdańsk, Kładki 24, 80-822 Gdańsk, Poland \\ ${ }^{\mathrm{b}}$ Department of Carbohydrate Chemistry, University of Gdańsk, Sobieskiego 18, 80-952 Gdańsk, Poland

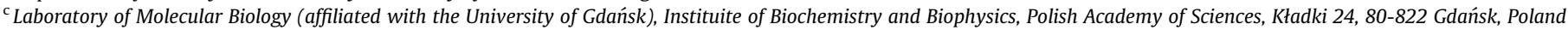 \\ ${ }^{\mathrm{d}}$ Department of Hypertension and Diabetology, Medical University of Gdańsk, Dębinki 7, 80-210 Gdańsk, Poland
}

\section{A R T I C L E I N F O}

Article history:

Received 7 May 2010

Accepted 25 July 2010

\begin{abstract}
S U M M A R Y
Mucopolysaccharidoses (MPS) are inherited metabolic disorders from the group of lysosomal storage diseases (LSD). They arise from mutations causing dysfunction of one of enzymes involved in degradation of glycosaminoglycans (GAGs) in lysosomes. Impaired degradation of these compounds results in their accumulation in cells and dysfunction of most tissues and organs of patients. If heparan sulfate (HS) is the sole or one of stored GAGs, brain functions are also affected. However, despite the fact that products of incomplete degradation of the same chemical, HS, are accumulated in brains of patients suffering from Hurler disease (MPS type I), Hunter disease (MPS type II), Sanfilippo disease (MPS type III) and Sly disease (MPS type VII), and obvious deterioration of brain functions occur in these patients, their behavior is considerably different between various types of MPS. Here we asked the question about biochemical reasons of these differences. We performed theoretical analysis of products of incomplete HS degradation that accumulate in tissues of patients diagnosed for these diseases. A correlation between chemical structures of incompletely degraded HS and behaviors of patients suffering from particular MPS types was found. We propose a hypothesis that particular chemical moieties occurring at the ends of incompletely degraded HS molecules may determine characteristic behavioral disturbances, perhaps due to chemical reactions interfering with functions of neurons in the brain. A possible experimental testing of this hypothesis is also proposed. If the hypothesis is true, it might shed some new light on biochemical mechanisms of behavioral problems occurring not only in MPS but also in some other diseases.
\end{abstract}

(c) 2010 Elsevier Ltd. All rights reserved.

\section{Introduction}

Mucopolysaccharidoses (MPS) are inherited metabolic diseases, from the group of lysosomal storage disorders (LSD), that occur panethnically with cumulative frequencies of all their types of about 1 per $40.000-50.000$ live births [1]. These diseases are caused by mutations in genes coding for enzymes involved in degradation of glycosaminoglycans (GAGs) (formerly called mucopolysaccharides). Their impaired hydrolysis leads to continuous accumulation and storage of these compounds in cells of patients, which results in a damage of the affected tissues, including the heart, respiratory system, bones, joints and central nervous system (CNS). MPS are usually fatal diseases (especially neuronopathic forms of MPS), with average expected life span of one or two dec-

\footnotetext{
* Corresponding author. Tel.: +48 58523 6308; fax: +48 585235501 .

E-mail address: wegrzyn@biotech.univ.gda.pl (G. Węgrzyn).
}

ades, though patients with milder forms can survive into adulthood [1].

On the basis of the nature of lacking or defective enzyme and the kind(s) of stored GAG(s), 11 types and subtypes of MPS are distinguished (Table 1). Neuronopathic forms of MPS are found in 7 out of 11 types and subtypes, and dysfunctions of CNS correlate with storage of heparan sulfate (HS), which together with dermatan sulfate (DS) and keratan sulfate (KS) is one of main GAGs (Table 1). Since MPS are progressive diseases, due to continuous accumulation of GAGs, patients deteriorate continuously in the course of these disorders, which reflects also cognitive abilities and behavior of patients suffering from the neuronopathic forms. Although prediction of severity and clinical progression of MPS is very difficult, even if biochemical and genetic data are available [2], recent studies suggested that consideration of two or more biochemical parameters may give significantly better results in assessment of MPS patients than attempting to make conclusion based on a single biomarker [3]. 
Table 1

Mucopolysaccharidoses (MPS), stored GAGs, deficient enzymes and neurological symptoms.

\begin{tabular}{|c|c|c|c|}
\hline MPS type (syndrome name) & $\begin{array}{l}\text { Main GAGs } \\
\text { stored in cells }^{\mathrm{a}}\end{array}$ & Deficient enzyme & Neurological symptoms \\
\hline $\begin{array}{l}\text { MPS I (Hurler disease - MPS I-H, Scheie disease - MPS I-S, } \\
\text { Hurler-Scheie disease - MPS I-H/S) }\end{array}$ & DS, HS & $\alpha$-L-iduronidase & $\begin{array}{l}\text { Severe (in the MPS I-H clinical subtype), very mild to mild } \\
\text { (in MPS I-H/S) or none (in MPS I-S) }\end{array}$ \\
\hline MPS II (Hunter disease) & DS, HS & Iduronate sulfatase & Severe or mild (rarely none) \\
\hline MPS IIIA (Sanfilippo disease subtype A) & HS & Heparan $N$-sulfatase & Severe \\
\hline MPS IIIB (Sanfilippo disease subtype B) & HS & $\alpha-N$-actylglucosaminidase & Severe \\
\hline MPS IIIC (Sanfilippo disease subtype C) & HS & $\begin{array}{l}\text { Acetyl-CoA: } \alpha- \\
\text { glycosaminide } \\
\text { acetyltransferase }\end{array}$ & Severe \\
\hline MPS IIID (Sanfilippo disease subtype D) & HS & $\begin{array}{l}\mathrm{N} \text {-acetylglucosamine } 6- \\
\text { sulfatase }\end{array}$ & Severe \\
\hline MPS IVA (Morquio disease subtype A) & KS & $\begin{array}{l}\mathrm{N} \text {-acetylgalactosaminide } 6 \text { - } \\
\text { sulfatase }\end{array}$ & None \\
\hline MPS IVB (Morquio disease subtype B) & KS & $\beta$-galactosidase & None \\
\hline MPS VI (Maroteaux-Lamy disease) & DS & $\begin{array}{l}\mathrm{N} \text {-acetylgalactosamine } 4 \text { - } \\
\text { sulfatase }\end{array}$ & None \\
\hline MPS VII (Sly disease) & DS, HS & $\beta$-glucuronidase & Usually mild \\
\hline MPS IX & Hyaluronan & Hyaluronidase & None $^{\mathrm{b}}$ \\
\hline
\end{tabular}

a Abbreviations: DS - dermatan sulfate, HS - heparan sulfate, KS - keratan sulfate.

b Only one patient has been described to date, thus, this case is non-conclusive for occurrence of neurological symptoms in this disease.

\section{Neuronopathy in mucopolysaccharidoses}

As shown in Table 1, most patients suffering from MPS I (Hurler disease), MPS II (Hunter diseases) and MPS VII (Sly diseases), and all patients suffering from MPS III (Sanfilippo disease) develop CNS pathology, which results not only in neurological symptoms but also in severe cognitive and behavioral disturbances. Among neurological problems of MPS patients, vision and hearing problems, epilepsy and various seizures may be recognized as predominant symptoms [1]. However, apart from such symptoms, neuronopathic forms of MPS are characterized by severe behavioral disturbances and loosing of cognitive skills [4-6]. In this paper we will focus on these behavioral disturbances as they are considerably different in various MPS types.

\section{Various behaviors in different MPS types}

Although loosing of cognitive skills is common in vast majority of neuronopathic MPS patients, there are obvious differences between behaviors of children affected with various MPS types. In MPS I patients with neuronopathy, despite problems with learning and cognitive deterioration, little or no behavioral problems occur $[1,5]$. Such a clinical picture is also common for MPS VII patients [1]. On the other hand, MPS II patients, and particularly MPS III patients (all subtypes), develop an aggressive behavior in the course of the disease [4-6]. MPS II children are often overactive, but this symptom is especially severe in MPS III patients, which are usually described as hyperactive. These patients have usually severe sleeping defects, and can sleep only a couple of hours a day. MPS II and MPS III children may become unexpectedly angry or may laugh without any reason. Contrary to them, MPS I and MPS VII patients are generally placid, gentle and calm, even if cognitive deterioration is severe. Furthermore, MPS I children are often over-careful, while MPS III patients appear to ignore any danger, and their behavior suggests intensive action without any particular sense, often leading to body injuries due to running into potentially dangerous environment.

These different behaviors, characteristic for particular MPS types and discussed in various review articles [1,4-6], are summarized in Table 2 . Although these behaviors are clinically clearly recognized, the reason of such huge differences between patients accumulating the same storage material, HS, is unclear.

\section{Potential biochemical reasons of various behaviors among patients suffering from different MPS types}

As it is evident from Tables 1 and 2, all neurological symptoms and behavioral disturbances which occur in MPS patients correlate with storage of HS. However, variety of behavioral problems characteristic for particular MPS types is surprising. One extreme is behavior of MPS I and MPS VII patients, which is generally normal and the affected children are usually placid, gentle and calm. Another extreme is behavior of MPS III patients, i.e. their hyperactivity, aggressive behavior and sleepless. Behavior of MPS II patients can be classified as intermediate, between these two extremes.

The above described differences cannot be explained on the basis of the old hypothesis on the mechanism of lysosomal storage diseases, which assumed that a storage of an undegraded material in lysosomes is the only cause of clinical symptoms. If this was the case, storage of various partially degraded HS derivatives should cause similar symptoms, which is clearly not true. More recent concept of the mechanism of neuropathology in MPS patients arose from the fact that there are secondary storage products, found in brains of both animal models and patients suffering from MPS, especially gangliosides GM2 (II3- N-acetylneuraminosylgangliotriaosylceramide) and GM3 (II3- $N$-acetylneuraminosyllactosylceramide), which are believed to be responsible for neurodegeneration in some other lysosomal storage diseases [7-16]. This secondary storage could be caused by GAG-mediated inhibition of activities of lysosomal enzymes involved in ganglioside degradation [17-19]. Interestingly, the presence of accumulated GM2 and GM3 gangliosides correlated with neuropathology in MPS. Therefore, as discussed recently [20], it was proposed that these secondary storage materials, rather than primarily stored GAGs, might be responsible for neuronopathy in MPS patients. However, GAGs and gangliosides were found to be localized differentially in MPS cells [13]. Moreover, accumulation of gangliosides was also observed in lysosomal storage diseases without either defects in enzymes involved directly in their degradation or know GAG storage [21]. Therefore, it was concluded that these results raise significant questions about whether GAG-induced inhibition of lysosomal enzymes occurs in vivo, and whether it is a mechanism underlying GM2 and GM3 storage in MPS [20]. Although these questions do not exclude a role for gangliosides in development of neuropathology during the course of MPS diseases, the secondary storage of these compounds definitely cannot explain the dif- 
Table 2

Behavioral disturbances in various types of MPS.

\begin{tabular}{|c|c|c|}
\hline $\begin{array}{l}\text { MPS } \\
\text { type }\end{array}$ & Characteristic behavior & $\begin{array}{l}\text { Additional (apart from those common for all carbohydrates) chemical moieties } \\
\text { at the ends of partially degraded HS }\end{array}$ \\
\hline MPS I & $\begin{array}{l}\text { Little or no behavioral problems; placid and calm behavior; sometimes } \\
\text { over-careful behavior }\end{array}$ & None \\
\hline MPS II & Aggressive behavior; overactivity; anger or laugh without a reason & Sulfate (bound directly to the carbohydrate backbone) \\
\hline $\begin{array}{l}\text { MPS III } \\
\qquad(A-D)\end{array}$ & $\begin{array}{l}\text { Aggressive behavior; hyperactivity; sleep disturbance; anger or laugh } \\
\text { without a reason; no care on danger }\end{array}$ & $N$-sulfate, amino, $N$-acetyl \\
\hline MPS VII & Little or no behavioral problems; placid and calm behavior & None \\
\hline
\end{tabular}

ferences in behaviors of patients suffering from various MPS types. Namely, the same kinds of gangliosides accumulate in all neuronopathic forms of MPS diseases [20], while behaviors of patients are considerably different.

It became more and more obvious that undegraded or partially degraded GAGs are not only stored in lysosomes, but they can also accumulate outside these organelles, and outside cells. As demonstrated recently, in a culture of human fibroblasts derived from an MPS patient, in the presence of factors allowing effective synthesis of GAGs, there are so many lysosomes fulfilled with the storage material, that some of them can be damaged or even "excreted" outside the cell [22]. It is likely that in vivo, the material from excreted and damaged lysosomes may then accumulate in the extracellular matrix and can interfere with reactions between cells. If this occurs in the brain, transmission of signals between neurons can be influenced by reactive small molecules or chemical moieties of larger molecules.

In the light of the assumption described above, it is necessary to consider the biochemical pathway of HS degradation. It is worth noting that this degradation proceeds gradually, by removing particular chemical moieties or monosaccharides, step by step. This degradation pathway, established on the basis of biochemical analyses performed in vitro, is depicted in Fig. 1. Inability to remove the sulfate moiety, due to deficiency of iduronate sulfatase, causes a block in further degradation of HS, and results in MPS II. A lack of $\alpha$-L-iduronidase activity causes MPS I, as iduronate cannot be removed from the end of the HS polysaccharide chain. Deficiency in next three enzymes in this pathway results in inhibition of HS degradation at subsequent steps, and is a cause of symptoms characteristic for MPS IIIA, $C$ and $B$, respectively. In fact, clinical symptoms of all four MPS III subtypes (A-D) are similar, and thus, these disorders are grouped in the one type of MPS (type III), called also Sanfilippo disease. It is interesting that a disease caused by deficiency in activity of the next enzyme in the HS degradation pathway, glucuronate sulfatase, is not known. Despite this, a lack of the $\beta$-glucuronidase causes MPS VII, and $N$-actylglucosamine 6-sulfatase deficiency results in symptoms of MPS IIID.

Importantly, specific biochemical markers, occurring in urine of patients, characteristic for particular MPS types and subtypes, were identified [23]. These markers consisted of oligosaccharides, which have a non-reducing terminus identical to the specific substrate for the deficient enzyme in the particular MPS disease. For example, di- and tri-saccharides characteristic for MPS I (the disease caused by deficiency of $\alpha$-L-iduronidase) contained $\alpha$-L-iduronic acid at the non-reducing end, oligosaccharides specific for MPS II (the disease caused by deficiency in iduronate sulfatase) did bear the sulfate on the terminal non-reducing $\alpha$-L-iduronic acid, and so on [23]. These results provided a proof that theoretically predicted (on the basis of biochemical analyses performed in vitro) products of incomplete degradation of GAGs occur in bodies of MPS patients, indeed. Most probably, the oligosaccharides detected experimentally in urine samples of patients arose from endolytic cleavage of larger GAG fragments followed by exolytic trimming to the resistant (due to a lack of particular enzymes) structures. From the point of view of mechanisms of neuronopathy in MPS diseases, another discov-

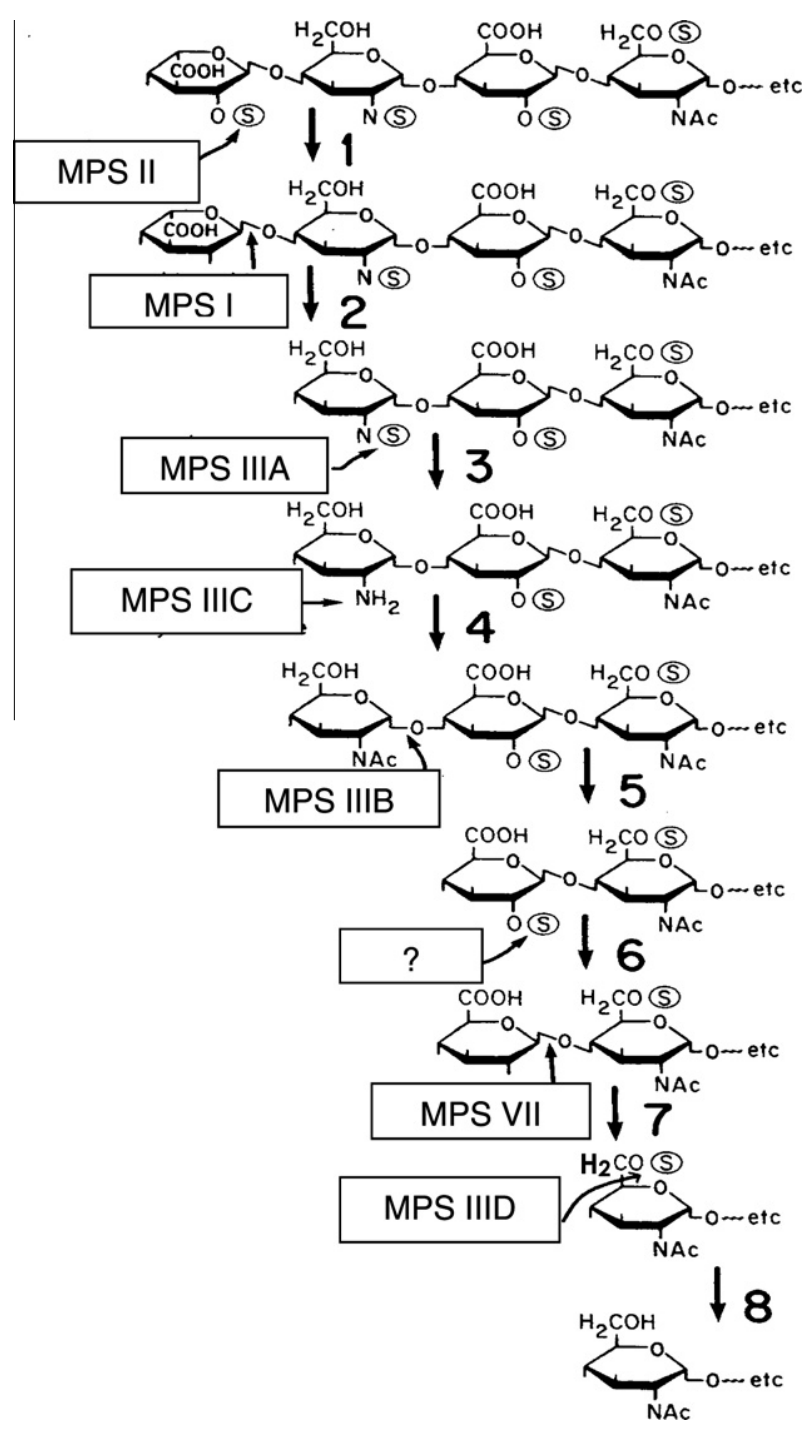

Fig. 1. The pathway of HS degradation. Particular degradation steps, marked by numbers, are catalyzed by enzymes whose deficiencies result in certain MPS types, shown in frames (a disease caused by deficiency of glucuronate sulfatase is not known, and is described as a question mark). The enzymes catalyzing particular reactions are as follows: 1 - iduronate sulfatase, $2-\alpha$-L-iduronidase, 3 - heparan $N$ sulfatase, 4 - acetyl-CoA: $\alpha$-glycosaminide acetyltransferase, $5-\alpha$ - $N$-actylglucosaminidase, 6 - glucuronate sulfatase, 7 - $\beta$-glucuronidase, 8 - N-acetylglucosamine 6 -sulfatase. The 'circled $S$ ' indicates the sulfate moiety. The scheme is based on the review by Neufeld and Muenzer [1], and was modified.

ery appears to be crucial. Namely, levels of di- and tri-saccharides characteristic for MPS I correlated with the presence/absence of CNS pathology in patients [24]. These results strongly suggest that chemical products of incomplete HS degradation, which occur in vivo, are responsible (either directly or indirectly) for development of CNS problems (including behavioral ones) in MPS patients. 
In the light of results described above, when looking for potential biochemical mechanism of differences in behaviors of patients suffering from various MPS types, one should ask a question whether it is possible to find any chemical features which are common for three groups of behavioral schemes, characteristic for: (i) MPS I and MPS VII, (ii) MPS III (A-D), and (iii) MPS II. Interestingly, in bodies of MPS patients who express little or no behavioral disturbances, i.e. MPS I and MPS VII patients, there is an accumulation of intermediates of HS degradation which contain no additional (i.e. apart from those which are sugar-specific $-\mathrm{OH}$ and $-\mathrm{H}$ ) chemical moieties at the non-reducing terminus (Fig. 1 and Table 2). On the other hand, MPS III correlates with storage of partially degraded HS molecules containing, at the non-reducing terminal monosaccharide, either $N$-sulfate (MPS IIIA), amino (MPS IIIC) or $\mathrm{N}$-acetyl (MPS IIIB and MPS IIID) moiety. MPS II patients, with the intermediate behavior, accumulate the compound bearing the sulfate moiety at the non-reducing terminal monosaccharide, which is, contrary to MPS IIIA, $\mathrm{O}$-bonded rather than $\mathrm{N}$-bonded.

On the basis of the above analysis, we suggest that the presence of the partially degraded HS with sulfate or acetyl moiety bonded to the sugar backbone through the $\mathrm{N}$ atom, or only the amino moiety, at the non-reducing terminus, causes behavioral symptoms characteristic for MPS III. We speculate that this might be due to reactivity of these $\mathrm{N}$-containing moieties. Moreover, if sulfate moiety is $O$-bonded to the non-reducing terminal monosaccharide of HS degradation intermediate, the MPS II-like behavior occurs. Finally, no additional chemical moieties at the non-reducing terminus of partially degraded HS results in no behavioral disturbances, as observed in MPS I and MPS VII patients. If this hypothesis were true, putative patients suffering from a currently unknown disease caused by deficiency in glucuronate sulfatase should develop a behavior similar to that described for MPS II (compare Fig. 1 and Table 2).

The above presented proposal is hypothetical, however, it opens clear ways to its experimental testing. Namely, if this hypothesis is true, behaviors of experimental animals whose brains were injected with either synthetic monosaccharides corresponding to non-reducing terminal sugars occurring in particular MPS types or oligosaccharides characteristic for particular MPS types and identified previously [23], should be similar to behaviors characteristic for MPS I, MPS II, MPS III and MPS VII patients. Moreover, such behaviors should correspond to those occurring in animal models of these diseases. It is worth mentioning that such models of MPS I, MPS II, MPS IIIA, MPS IIIB and MPS VII are already available [25-32].

Finally, if the presented hypothesis were true, it might shed a new light on biochemical mechanisms of behavioral disturbances found not only in MPS but also in other diseases. Namely, specific chemical moieties might influence particular reactions occurring in brain during transmission of signals between neurons. In fact, although direct molecular reasons for abnormal behaviors of affected patients remain to be elucidated, it was indicated recently that results of studies on neuronopathic MPS and other LSD may be very important to understand pathomechanisms of many other neurodegenerative disorders, including Alzheimer disease, Parkinson disease and Huntington disease [33].

\section{Conflicts of interest statement}

None Declared.

\section{Acknowledgments}

This work was supported by Ministry of Science and Higher Education of Poland (Project Grants No. N302 046 32/3603 to
G.W. and 3631/B/P01/2007/33 to J.J.-B.), and was operated within the Foundation for Polish Science Team Programme co-financed by the EU European Regional Development Fund (Grant No. TEAM/2008-2/7 to G.W.).

\section{References}

[1] Neufeld EF, Muenzer J. The mucopolysaccharidoses. In: Scriver CR, Beaudet AL, Sly WS, Valle D, editors. The metabolic and molecular bases of inherited disease. New York: McGraw-Hill; 2001. p. 3421-52.

[2] Wegrzyn G, Wegrzyn A, Tylki-Szymanska A. A general model for genetic regulation of turnover of glycosaminoglycans suggests a possible procedure for prediction of severity and clinical progress of mucopolysaccharidoses. Med Hypotheses 2004;62(6):986-92.

[3] Piotrowska E, Jakobkiewicz-Banecka J, Tylki-Szymanska A, Czartoryska B, Wegrzyn A, Wegrzyn G. Correlation between severity of mucopolysaccharidoses and combination of the residual enzyme activity and efficiency of glycosaminoglycan synthesis. Acta Paediatr 2009;98(4):743-9.

[4] Al Sawaf S, Mayatepek E, Hoffmann B. Neurological findings in Hunter disease: Pathology and possible therapeutic effects reviewed. J Inherit Metab Dis 2008;31(4):473-80.

[5] Terlato NJ, Cox GF. Can mucopolysaccharidosis type I disease severity be predicted based on a patient's genotype? A comprehensive review of the literature. Genet Med 2003;5(4):286-94.

[6] Valstar MJ, Ruijter GJ, van Diggelen OP, Poorthuis BJ, Wijburg FA. Sanfilippo syndrome: A mini-review. J Inherit Metab Dis 2008.

[7] Bermudez AJ, Johnson GC, Vanier MT, Schroder M, Suzuki K, Stogsdill PL, et al. Gangliosidosis in emus (Dromaius novaehollandiae). Avian Dis 1995;39(2): 292-303.

[8] Constantopoulos G, Dekaban AS. Neurochemistry of the mucopolysaccharidoses: brain lipids and lysosomal enzymes in patients with four types of mucopolysaccharidosis and in normal controls. J Neurochem 1978;30(5): 965-73.

[9] Constantopoulos G, Iqbal K, Dekaban AS. Mucopolysaccharidosis types IH, IS, II, and IIIA: glycosaminoglycans and lipids of isolated brain cells and other fractions from autopsied tissues. J Neurochem 1980;34(6):1399-411.

[10] Constantopoulos G, Shull RM, Hastings N, Neufeld EF. Neurochemical characterization of canine alpha-L-iduronidase deficiency disease (model of human mucopolysaccharidosis I). J Neurochem 1985;45(4):1213-7.

[11] Hara A, Kitazawa N, Taketomi T. Abnormalities of glycosphingolipids in mucopolysaccharidosis type III B. J Lipid Res 1984;25(2):175-84.

[12] Li HH, Yu WH, Rozengurt N, Zhao HZ, Lyons KM, Anagnostaras S, et al. Mouse model of Sanfilippo syndrome type B produced by targeted disruption of the gene encoding alpha-N-acetylglucosaminidase. Proc Natl Acad Sci U S A 1999;96(25):14505-10.

[13] McGlynn R, Dobrenis K, Walkley SU. Differential subcellular localization of cholesterol, gangliosides, and glycosaminoglycans in murine models of mucopolysaccharide storage disorders. J Comp Neurol 2004;480(4):415-26.

[14] Russell C, Hendson G, Jevon G, Matlock T, Yu J, Aklujkar M, et al. Murine MPS I: Insights into the pathogenesis of Hurler syndrome. Clin Genet 1998;53(5): 349-61.

[15] Siegel DA, Walkley SU. Growth of ectopic dendrites on cortical pyramidal neurons in neuronal storage diseases correlates with abnormal accumulation of GM2 ganglioside. J Neurochem 1994;62(5):1852-62.

[16] Van Dessel G, Lagrou A, Martin JJ, Ceuterick C, Dierick W. Two cases of mucopolysaccharidosis type III (Sanfilippo). A biochemical study. J Neurol Sci 1979;40(2-3):77-86.

[17] Avila JL, Convit J. Inhibition of leucocytic lysosomal enzymes by glycosaminoglycans in vitro. Biochem J 1975;152(1):57-64.

[18] Baumkotter J, Cantz M. Decreased ganglioside neuraminidase activity in fibroblasts from mucopolysaccharidosis patients. Inhibition of the activity in vitro by sulfated glycosaminoglycans and other compounds. Biochim Biophys Acta 1983;761(2):163-70.

[19] Kint JA, Dacremont G, Carton D, Orye E, Hooft C. Mucopolysaccharidosis: secondarily induced abnormal distribution of lysosomal isoenzymes. Science 1973;181(97):352-4.

[20] Walkley SU, Vanier MT. Secondary lipid accumulation in lysosomal disease. Biochim Biophys Acta 2009;1793(4):726-36.

[21] Walkley SU. Secondary accumulation of gangliosides in lysosomal storage disorders. Semin Cell Dev Biol 2004;15(4):433-44.

[22] Jakobkiewicz-Banecka J, Piotrowska E, Narajczyk M, Baranska S, Wegrzyn G. Genistein-mediated inhibition of glycosaminoglycan synthesis, which corrects storage in cells of patients suffering from mucopolysaccharidoses, acts by influencing an epidermal growth factor-dependent pathway. J Biomed Sci 2009;16:26.

[23] Fuller M, Rozaklis T, Ramsay SL, Hopwood JJ, Meikle PJ. Disease-specific markers for the mucopolysaccharidoses. Pediatr Res 2004;56(5):733-8.

[24] Fuller M, Brooks DA, Evangelista M, Hein LK, Hopwood JJ, Meikle PJ. Prediction of neuropathology in mucopolysaccharidosis I patients. Mol Genet Metab 2005;84(1):18-24.

[25] Bhattacharyya R, Gliddon B, Beccari T, Hopwood JJ, Stanley P. A novel missense mutation in lysosomal sulfamidase is the basis of MPS III A in a spontaneous mouse mutant. Glycobiology 2001;11(1):99-103. 
[26] Bhaumik M, Muller VJ, Rozaklis T, Johnson L, Dobrenis K, Bhattacharyya R, et al. A mouse model for mucopolysaccharidosis type III A (Sanfilippo syndrome). Glycobiology 1999;9(12):1389-96.

[27] Birkenmeier EH, Davisson MT, Beamer WG, Ganschow RE, Vogler CA, Gwynn B et al. Characterization of a mouse with beta-glucuronidase deficiency. J Clin Invest 1989;83(4):1258-66.

[28] Clarke LA, Russell CS, Pownall S, Warrington CL, Borowski A, Dimmick $\mathrm{JE}$, et al. Murine mucopolysaccharidosis type I: Targeted disruption of the murine alpha-L-iduronidase gene. Hum Mol Genet 1997;6(4): 503-11.

[29] Haskins ME. Animal models for mucopolysaccharidosis disorders and their clinical relevance. Acta Paediatr Suppl 2007;96(455):56-62.
[30] Muenzer J, Lamsa JC, Garcia A, Dacosta J, Garcia J, Treco DA. Enzyme replacement therapy in mucopolysaccharidosis type II (Hunter syndrome): a preliminary report. Acta Paediatr Suppl 2002;91(439):98-9.

[31] Sands MS, Birkenmeier EH. A single-base-pair deletion in the betaglucuronidase gene accounts for the phenotype of murine mucopolysaccharidosis type VII. Proc Natl Acad Sci U S A 1993;90(14):6567-71.

[32] Wang D, Shukla C, Liu X, Schoeb TR, Clarke LA, Bedwell DM, et al. Characterization of an MPS I-H knock-in mouse that carries a nonsense mutation analogous to the human IDUA-W402X mutation. Mol Genet Metab 2010;99(1):62-71.

[33] Bellettato CM, Scarpa M. Pathophysiology of neuropathic lysosomal storage disorders. J Inherit Metab Dis 2010; (DOI: 10.1007/s10545-010-9075-9). 